\title{
INFRASTRUCTURE SYSTEMS FOR INTERSECTION COLLISION AVOIDANCE
}

\author{
Robert A. Ferlis \\ Office of Operations Research and Development \\ Federal Highway Administration \\ McLean, Virginia USA \\ E-mail: robert.ferlis@fhwa.dot.gov
}

\begin{abstract}
Summary: This paper will describe conceptual outlines of possible infrastructure intersection collision avoidance systems. The infrastructure concepts represent countermeasures for crossing path crashes at intersections. Crossing path crashes involve one vehicle cutting across the path of another, both initially traveling from either perpendicular or opposite directions, in such a way that they collide. Infrastructure-based intersection collision avoidance systems use roadside sensors, processors, and warning devices; roadside-vehicle communication devices; and traffic signals to provide driving assistance to motorists.
\end{abstract}

Intersection safety has begun to receive new attention from traffic engineers, human factors specialists, and others who see that emerging intelligent systems offer significant potential for improvements (Ferlis, 1999). Crossing path crashes at intersections, as defined by Volpe (Barr, 2001), involve one vehicle cutting across the path of another, both initially traveling from either perpendicular or opposite directions, in such a way that they collide. Infrastructure-based intersection collision avoidance systems use roadside sensors, processors, and warning devices; roadside-vehicle communication devices; other roadside informational or warning devices; and traffic signals to provide driving assistance to motorists. The intersection collision avoidance systems can be classified as either infrastructure-only or as infrastructure vehicle cooperative. Infrastructure-only systems rely solely on roadside warning devices to communicate with drivers. Cooperative systems communicate information directly to vehicles and drivers. Major advantages of cooperative systems lie in their capabilities to improve the interface to the driver, and hence to virtually ensure that a warning is received. This could also take advantage of the potential to exert control over the vehicle, at least in situations where the system can be confirmed as reliable and the driver cannot reasonably be expected to take appropriate actions given the imminent hazard and response time available.

The investments in roadside sensing and processing of infrastructure-based cooperative systems will require only a minimal amount of in-vehicle equipment, which will include: (1) a communications transceiver, possibly based on Dedicated Short Range Communication (DSRC) technology; (2) an in-vehicle processor; and (3) the driver interface. These functions could be provided through aftermarket devices that might be installed by the motorist on the vehicle's windshield or dashboard, similar to a radar detector. Such a device might only cost \$50 in volume. Alternatively, vehicle manufacturers could incorporate these functions directly at time of manufacture. 


\section{SYSTEM CONCEPTS}

Four infrastructure intersection collision avoidance system concepts are described here.

\section{Traffic Signal Violation Warning}

This countermeasure involves (1) warning potential violators of a traffic signal to recognize the control device and (2) warning motorists on adjacent approaches of the potential conflict. The target crashes reflect causal factors of "did not see”, “ tried to beat the light”, or "deliberate violation”, for a total of 288,000 crashes per year (Barr, 2001).

The basic sensing requirements are to identify potential (very highly likely) violators by determining the speed and possibly also the deceleration rate of each vehicle at a fixed location. Sensing of speed at a point can readily be accomplished with conventional magnetic "loop" detectors, self-powered vehicle detectors (SPVD), optical sensors, or radar sensors. Sensing systems capable of measuring deceleration would be more expensive, but could significantly reduce the number of false detections.

The processing system would identify vehicles at an upstream control point that are unlikely to stop at the intersection. Preliminary calculations suggest, for example, that a vehicle traveling 30 mph at a point 100 feet upstream from the stop line will very likely be incapable of stopping in time (at least without a severe braking event), and hence can be identified as a potential violator. The processing logic will likely be incorporated in an Advanced Traffic Controller (ATC), which might represent an upgrade to the signal controller at the intersection. The ATC would operate the relatively simple algorithm for detecting potential violators, and would also monitor the sensors and control the roadside warning devices and any communication equipment (for a future cooperative system).

Once a violator is identified, warnings will be conveyed to the violator and also to other drivers on adjacent approaches to the intersection. The violator could be warned by: (1) Warning signs and lights activated once the potential violation is detected. For example, "Stop Ahead" warning signs could be used with a flashing amber light to draw attention to the signs, and located on both sides of the roadway to increase the likelihood that the subject driver would readily receive the warning. (2) A warning light could be incorporated directly in the traffic signal display itself, again to draw attention to the traffic control device. For example, strobe lights have been used to heighten the conspicuity of traffic signal displays for rural intersections where motorists may not expect a signal. (3) An intelligent rumble strip could be activated to warn the violator to slow down, and possibly heighten awareness of the need to stop at the intersection. (4) Variable message signs (VMS) could convey the warning to the driver.

Motorists on adjacent approaches also need to be warned of the potential violation and conflict, and could be warned by: (1) Warning lights activated to indicate a need for caution and possibly to indicate the source of the conflict. (2) An intelligent rumble strip activated to warn the other motorists to slow down and proceed cautiously at the intersection. (3) A VMS or graphic display sign used to warn drivers of the potential conflict with the signal violator, but these might only be effective only where there is sufficient time for the motorists to comprehend the message and respond. 
The costs for specific components needed for an infrastructure-only system are estimated as: (1) Sensors, at \$4,000 per approach. (2) Controller upgrade, at \$3,000. (3) Warning lights for violators (two per approach) and other drivers (four per approach), at \$200 per light. If engineering and other construction costs add $\$ 8,000$, the total costs per intersection can be estimated as $\$ 31,800$. Costs for a cooperative system would also include the roadside transceivers and antennas, and would increase the total costs for an intersection system to $\$ 57,800$, exclusive of in-vehicle equipment costs.

\section{Stop Sign Violation Warning}

This countermeasure involves (1) warning potential violators of a stop sign to recognize the control device and (2) warning motorists on adjacent approaches of the potential conflict. The target crashes reflect causal factors of “did not see” or “deliberate violation”, for a total of 79,000 crashes per year (Barr, 2001).

The basic sensing and processing requirements are similar to those previously described for traffic signal violations, except that the processing system would likely consist of a specialized controller, since a standard traffic signal controller will not be available.

Once a violator is identified, warnings will be conveyed to the violator and also to other drivers on adjacent approaches to the intersection. The violator could be warned by: (1) Warning signs and lights activated once the potential violation is detected. (2) A warning light activated adjacent to the "Stop" sign itself, again to draw attention to the traffic control device. (3) An intelligent rumble strip activated to warn the violator to slow down, and possibly heighten awareness of the need to stop at the intersection.

Motorists on adjacent approaches could be warned by: (1) Warning lights to indicate a need for caution and the source of the conflict. (2) A VMS to warn drivers of the potential conflict with the stop sign violator. (3) An intelligent rumble strip.

The costs for specific components needed for an infrastructure-only system are estimated as: (1) Sensors, at \$2,000 per approach (for 6 x 6 foot loops or cameras). (2) Controller and cabinet, at $\$ 4,000$. (3) Warning lights for violator (one per approach) and other drivers (four per approach), at $\$ 200$ per light. If engineering and other construction costs add $\$ 5,000$, the total costs per intersection can be estimated as $\$ 21,000$. Costs for a cooperative system would also include the roadside transceivers and antennas, and would increase the total costs for an intersection system to $\$ 47,000$.

\section{Traffic Signal Left Turn Assistance}

This countermeasure involves warning motorists making a left turn at a traffic signal of a potential conflict with vehicles approaching from the opposite direction. The target crashes reflect an "insufficient gap” causal factor, with a total of 192,000 crashes per year (Barr, 2001).

The basic sensing requirements are to identify potential conflicts by determining the speed and the acceleration or deceleration rate of each vehicle approaching the intersection from the opposite direction, including vehicles executing through and right turn movements. Simple point 
measurements will not be sufficient, since vehicles can assume various trajectories and acceleration/deceleration/stopping movements, particularly when other vehicles are present. As a consequence, the sensing of speed and acceleration/deceleration must be accomplished either with vision-based or radar sensors that can operate over a sufficiently broad field of regard.

The processor would determine whether each vehicle approaching from the opposite direction is likely to conflict with a left turn movement by the subject vehicle. The processing system would likely require an ATC, which might represent an upgrade to the signal controller at the intersection.

This countermeasure is presumed to apply to intersections with permissive left turn phasing only, and would convey whether a left turn can be safely initiated. Although the use of supplemental VMS or other signals could conceivably be considered, a more obvious warning device is the left turn arrow, modified for its new use. This application may particularly benefit from cooperative communication, since an in-vehicle system should have many advantages over roadside intervention methods.

The costs for specific components for infrastructure-only systems are estimated as: (1) Sensors, at $\$ 6,000$ per approach. (2) Controller upgrade, at $\$ 3,000$. (3) Turn arrow lamps, two per approach, at $\$ 500$. If engineering and other construction costs add $\$ 8,000$, the total costs per intersection can be estimated as $\$ 39,000$. Costs for a cooperative system would also include the roadside transceivers and antennas, and would increase the total costs for an intersection system to $\$ 65,000$.

\section{Stop Sign Movement Assistance}

This countermeasure involves warning motorists discharging from a stop sign that their movement may conflict with another vehicle. The subject vehicle movement could be left turn, right turn, or through. The target crashes reflect an "insufficient gap" causal factor, for a total of 362,000 crashes per year (Barr, 2001).

The basic sensing requirements are to identify potential conflicts by determining the speed, acceleration, or deceleration rate of each vehicle approaching the intersection and the discharge from the stop line for vehicles at stop-controlled approaches. The sensing of speed and acceleration/deceleration must be accomplished either with vision-based or radar sensors that can operate over a sufficiently broad field of regard.

The processor would determine whether each vehicle approaching the intersection is likely to conflict with a movement by the subject vehicle as it discharges from the stop line. The processing system would likely require a specialized controller. The controller would operate the algorithm for determining safe discharge of vehicles, would monitor the sensors, and would control the roadside warning devices and any communication equipment (for a future cooperative system).

This countermeasure could potentially be implemented either with VMS or else with graphical display signs, as was used successfully in the recent prototype Collision Countermeasure System installed in Prince William County, Virginia (Hanscom, 2001). Another possible approach is to 
develop a relatively simple signal that can convey permissible movements to drivers as they consider their movement from the stop line.

The costs for specific components for an infrastructure-only system are estimated as: (1) Sensors, at $\$ 6,000$ per approach. (2) Controller and cabinet, at $\$ 4,000$. (3) VMS or graphical signs, with one per approach at $\$ 3000$. If engineering and other construction costs add $\$ 5,000$, the total costs per intersection can be estimated as $\$ 45,000$. Costs for a cooperative system would also include the roadside transceivers and antennas, and would increase the total costs for an intersection system to $\$ 71,000$.

\section{DEPLOYMENT POTENTIAL}

One area for further exploration is the likely evolution of infrastructure-only systems into infrastructure vehicle cooperative systems. The deployment path for these systems is likely to require the provision of infrastructure-only systems first, with cooperative systems provided after a significant deployment of infrastructure-only systems has been achieved. This model assumes that vehicle manufacturers and aftermarket suppliers of in-vehicle systems will be reluctant to develop and market the in-vehicle components until motorists are able to take advantage of their investments in the in-vehicle components. However, recent interest by representatives of the vehicle manufacturing industry in the potential for cooperative intersection collision avoidance systems suggests that an earlier deployment of cooperative systems might nevertheless be possible. An analysis of the possible impacts of these collision avoidance systems (Ferlis, 1999) suggested that the systems will likely be installed first at intersections with relatively poor safety records, so the proportion of crashes avoided may greatly exceed the proportion of intersections improved. This should further justify early deployment of these innovative systems.

\section{REFERENCES}

Barr, L.C., daSilva, M.P., and Hitz, J.S. Safety Benefits/Cost Assessment of Potential IVI Safety Systems. HW00Q Technical Information Exchange. Volpe National Transportation Systems Center. September 2000.

Ferlis, R. Intelligent Transportation Systems, Analysis of Infrastructure-Based System Concepts, Intersection Collision Avoidance Problem Area. Federal Highway Administration, U.S. Department of Transportation. December 1999.

Hanscom, F.R. Evaluation of the Prince William County Collision Countermeasure System. Virginia Transportation Research Council, Virginia Department of Transportation. February 2001. 